\title{
Analytical solution to the one-dimensional non-uniform absorption of solar radiation in uncoated and coated single glass panes
}

\author{
Miguel Hermanns ${ }^{\mathrm{a}, \mathrm{d}, *}$, Fernando del Ama ${ }^{\mathrm{b}, \mathrm{d}}$, Juan Antonio Hernández ${ }^{\mathrm{c}, \mathrm{d}}$ \\ a Dpto de Motopropulsión y Termofluidodinámica, Universidad Politécnica de Madrid, Plaza Cardenal Cisneros 3, 28040 Madrid, Spain

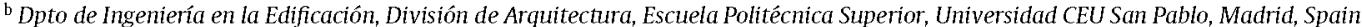 \\ ' Dpto de Matemática Aplicada y Estadística, Universidad Politécnica de Madrid, Ptaza Cardenal Cisneros 3, 28040 Madrid, Spain \\ ${ }^{\mathrm{d}}$ IntelliGlass S.L., Avda M-40 9 Bajo 11, Polígono Industrial Ventorro del Cano, 28925 Alcorcón, Spain
}

\begin{abstract}
A B S T R A C T
The analytical solution to the one-dimensional absorption-conduction heat transfer problem inside a single glass pane is presented, which correctly takes into account all the relevant physical phenomena: the appearance of multiple reflections, the spectral distribution of solar radiation, the spectral dependence of optical properties, the presence of possible coatings, the non-uniform nature of radiation absorption, and the diffusion of heat by conduction across the glass pane. Additionally to the well established and known direct absorptance $\alpha_{e}$, the derived solution introduces a new spectral quantity called direct absorptance moment $\beta_{e}$, that indicates where in the glass pane is the absorption of radiation actually taking place. The theoretical and numerical comparison of the derived solution with existing approximate thermal models for the absorption-conduction problem reveals that the latter ones work best for low-absorbing uncoated single glass panes, something not necessarily fulfilled by modern glazings.
\end{abstract}

\section{Introduction}

In recent years, the construction of buildings with extensive glass facades has increased rapidly. The widespread use of glass as facade elements represents a serious challenge for the heating and cooling of these buildings, since glazings poorly insulate from the cold exterior in wintertime and from the solar radiation in summertime. This fact increases the energy consumption of the building, its HVAC costs, and its environmental impact.

To address this problem, advanced glazings and well designed glass facades are required, which need to be analyzed a priori in order to ensure the low energy consumption of the building while keeping comfortable thermal conditions in its interior. The increasing complexity of the configurations to analyze and the increased requirements in energy savings lead to the use of advanced simulation tools with growing complexity and accuracy in the implemented models and algorithms.

However, although three-dimensional multi-physics simulations of complex glazings are possible nowadays [1-3], their

\footnotetext{
* Corresponding author at: Dpto de Motopropulsión y Termofluidodinámica, Universidad Politécnica de Madrid, Plaza Cardenal Cisneros 3, 28040 Madrid, Spain. Fax: +34913363295

E-mail address: miguel.hermanns@upm.es (M. Hermanns).
}

computational time is too high for applications like annual energy consumption studies of individual glazings [4,5] or of whole buildings with hundreds or even thousands of glazing units [6]. Therefore, accurate and efficient one-dimensional models for complex glazings are still necessary, which are easy and fast to evaluate, but still deliver accurate results.

All one-dimensional center-of-glass energy models for glazings are comprised by four main building blocks. The first one deals with the outer weather conditions, modeling things like the characteristics of the incoming radiation [7,8], the exterior temperature and wind conditions $[9,10]$, or the ground albedo $[11,12]$. The second one deals with the interaction of the radiation with the glazing, usually by solving the multiple reflections problem that arises, to determine the fractions of radiation that pass through the glazing and that are absorbed at the different glass panes of the glazing [13-15]. The third one deals with the absorption-conduction problem that takes place inside each of the glass panes [16-18]. And the fourth one deals with the subsequent heat transfer problem that arises between the different glass panes and between the glass panes and the exterior and interior environments, either through natural/forced convection or thermal radiation [19,20].

The present work focuses on the third building block: the absorption-conduction problem that takes place inside the individual glass panes of the glazing. Several physical phenomena need to be taken into account to correctly obtain the spatial absorption 
distribution across the glass pane and the resulting temperature profile, namely the appearance of multiple reflections, the spectral distribution of solar radiation, the spectral dependence of optical properties, the presence of possible coatings, the non-uniform nature of the radiation absorption process itself, and the diffusion of heat by conduction across the glass pane. Although all these physical phenomena are well known, almost all of the existing models for the absorption-conduction problem neglect some of them.

The two most extended simplifications are the constant temperature one [19,21,22] and the uniform absorption one [6,20,23-27]. The former neglects the thermal conduction problem inside the glass pane, while the latter approximates the non-uniform absorption of the incoming radiation by a uniform absorption distribution across the glass pane. Both simplifications work best for thin and low absorbing glass panes, two conditions not necessarily fulfilled by modern glazings. Additionally, the localized absorption introduced by applied coatings is not correctly taken into account by any of these two models, leading to additional errors [17].

In recent years, models for the absorption-conduction problem which take into account the non-uniform absorption phenomenon are being proposed. However, in their quest for analytical or simple expressions, most of them make simplifications in the way radiation is absorbed by the glass pane. The most common one is to neglect, completely or in part, the spectral dependence of the optical properties of the materials and of the spectral distribution of the incoming radiation $[1-3,18,28-33]$. Another common simplification is to neglect, completely or in part, the multiple reflections phenomenon that arises due to the presence of multiple material interfaces [1-3,28-33]. Only Rosenfeld [16] solves analytically the complete absorption-conduction problem with all the relevant physical phenomena. However, his approach is very much focused on the determination of the so-called secondary heat transfer factor towards the inside $[21,22]$, and is therefore not well suited for its inclusion in advanced simulation tools like WINDOW [23], VISION [24], or EnergyPlus [6], or in future revisions of the international standard ISO 15099 [20].

In the present work the one-dimensional absorptionconduction problem with all the relevant physical phenomena is also solved. However, the present approach leads to two algebraic equations which are easy to interpret, fast to solve, and can directly be implemented into existing simulation tools thanks to its structural similarity with existing approximate models. Along with the well known direct absorptance $\alpha_{e}$, a new spectral quantity called direct absorptance moment $\beta_{e}$ emerges from the equations, which indicates where in the glass pane is the absorption of radiation actually taking place, and its value is easily computed out of the solution to the multiple reflections problem and the spectral properties of the glass pane material, just like the value of $\alpha_{e}$. Thus, the replacement of existing approximate models for the absorption-conduction problem by the one presented here is simple and straightforward.

The paper is organized as follows. Section 2 presents the complete one-dimensional absorption-conduction heat transfer problem for a single glass pane, which is then solved analytically in Section 3 leading to a set of two algebraic equations. To better understand the resulting expressions, the limiting cases of weakly and strongly absorbing glass panes are analyzed in Section 4, while Sections 5 and 6 incorporate into the formulation the effects of possible coatings applied to the surfaces of the glass pane and of non-normal incidence angles of the radiation, respectively. Next, a review of existing approximate models for the absorption-conduction problem is presented in Section 7 , followed by a quantitative assessment of the accuracy of these approximate models in Section 8. Finally, the concluding remarks are presented in Section 9.

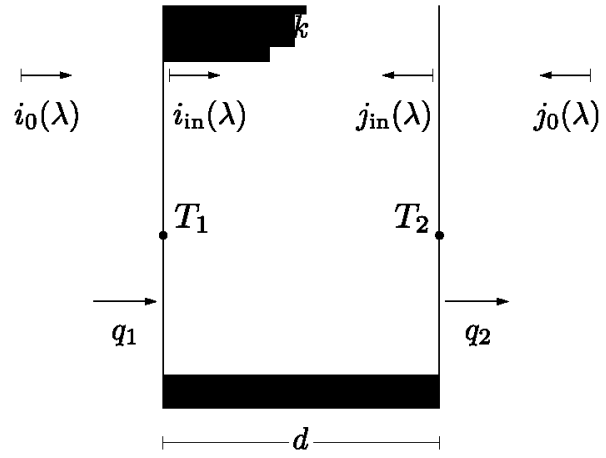

Fig. 1. Sketch of the absorption-conduction problem that arises from the interac tion of a glass pane of thickness $d$ and thermal conductivity $k$ with the incoming radiations $i_{0}(\lambda)$ and $j_{0}(\lambda)$ from the exterior and interior of the building, respectively.

\section{Formulation of the thermal problem}

Consider the glass pane of thickness $d$ and thermal conductivity $k$ depicted in Fig. 1, which represents either a single glazing or a glass pane that is part of a more complex glazing configuration like a laminated glass pane, a double or triple glazing, etc. From the left side, the solar radiation $i_{0}$ shines onto the glazing with a normal incidence angle and with a certain spectral distribution $i_{0}(\lambda)$, where $\lambda$ represents the radiation's wavelength. From the right side, another radiation $j_{0}$ with a different spectral distribution $j_{0}(\lambda)$ shines also onto the glazing with a normal incidence angle, being its origin the radiation reflected from the interior of the building, the radiation emitted by interior light sources, etc.

The interaction of the radiation with the glazing and its multiple material interfaces leads to a multiple reflections problem that needs to be solved in order to determine the fractions of radiation that are reflected, transmitted, and absorbed by the glazing. This is accomplished by using any of the methods belonging to the second group of main buildings blocks of energy models for glazings, like the ray tracing method [34,35], the matrix method $[13,14]$, or the net energy balance method $[20,36]$. The outcome of any of these methods are the four functions $A(\lambda), B(\lambda), C(\lambda)$, and $D(\lambda)$ which relate the radiation inside the considered glass pane with the incoming radiations $i_{0}(\lambda)$ and $j_{0}(\lambda)$ in the following way:

$$
i_{\text {in }}(\lambda)=A(\lambda) i_{0}(\lambda)+B(\lambda) j_{0}(\lambda)
$$

$j_{\text {in }}(\lambda)=C(\lambda) i_{0}(\lambda)+D(\lambda) j_{0}(\lambda)$

The ray $i_{\text {in }}(\lambda)$ is the radiation encountered just at the right of the left boundary of the glass pane and which travels to the right. The ray $j_{\text {in }}(\lambda)$ is the radiation encountered just at the left of the right boundary of the glass pane and which travels to the left. See Fig. 1 for a graphical explanation of $i_{\text {in }}(\lambda)$ and $j_{\text {in }}(\lambda)$.

The absorption by the glass pane of these right and left traveling radiations is given by Bouguer's Law [35]:

$q_{\text {rad }, \lambda}(x, \lambda)=a(\lambda)\left[i_{\text {in }}(\lambda) e^{-a(\lambda) x}+j_{\text {in }}(\lambda) e^{-a(\lambda)(d-x)}\right]$

where $q_{\operatorname{rad}, \lambda}(x, \lambda)$ is the energy per unit volume, unit time, and unit wavelength absorbed at the location $x$ inside the glass pane and at the radiation wavelength $\lambda$, and $a(\lambda)$ is the absorption coefficient of the material, which is a function of wavelength. In order to obtain the total amount of radiation energy $q_{\text {rad }}(x)$ absorbed per unit volume and unit time at a given location $x$, the contribution from all wavelengths of the incoming radiation needs to be taken into account by integrating $q_{\mathrm{rad}, \lambda}(x, \lambda)$ over the whole wavelength spectrum:

$q_{\text {rad }}(x)=\int_{0}^{\infty} q_{\text {rad }, \lambda}(x, \lambda) \mathrm{d} \lambda$ 
Next, the absorbed heat at each location $x$ is diffused through thermal conduction across the glass pane. Since the thickness $d$ is small compared to the typical size of the window, and the outer and inner conditions vary slowly in comparison to the thermal response time of the glass pane, the resulting absorption-conduction thermal problem can be considered quasi-steady and one-dimensional, thus leading to the following simplified energy conservation equation:

$0=\frac{d}{d x}\left(k \frac{d T}{d x}\right)+q_{\text {rad }}(x)$

Two boundary conditions are required to solve this second order differential equation. Depending on the position of the considered glass pane inside the glazing, these would be either the continuity of temperatures and heat fluxes with neighboring material layers or the heat fluxes imposed by the exposure of the glazing to the exterior and interior environments. From the point of view of the absorption-conduction problem itself, the actual nature of the boundary conditions is irrelevant, and the following general case will be considered here:

$\begin{array}{ll}x=0: & T=T_{1}, \quad-k \frac{d T}{d x}=q_{1} \\ x=d: & T=T_{2}, \quad-k \frac{d T}{d x}=q_{2}\end{array}$

Since only two boundary conditions can actually be imposed, the other two quantities introduced in the above relations are in fact a result to be obtained from the solution to the absorption-conduction problem.

\section{Solution to the thermal problem}

When solving the above one-dimensional absorptionconduction problem, it is important to bear in mind the final aim of the analysis: the determination of temperatures and heat fluxes at the surfaces of the glass pane. Therefore, it would be desirable to derive relations between the temperatures and heat fluxes at both sides of the glass pane without needing to look into the details of what is happening inside it.

To accomplish this, Eq. (4) is integrated once, and the heat flux $q_{1}$ is imposed at the left boundary of the glass pane, leading to

$-k \frac{d T}{d x}-q_{1}=\int_{0}^{x} q_{\text {rad }}(\xi) \mathrm{d} \xi$

Integrating again and imposing that the temperature at the left boundary is $T_{1}$ leads to

$-k\left(T-T_{1}\right)-q_{1} x=\int_{0}^{x} \int_{0}^{\eta} q_{\mathrm{rad}}(\xi) \mathrm{d} \xi \mathrm{d} \eta$

where it has been assumed that the thermal conductivity $k$ is constant across the glass pane. This assumption is reasonable since temperature variations across glass panes are usually small compared to the temperatures themselves, and so are the possible variations of the thermal conductivity.

Particularizing now Eqs. (6) and (7) at $x=d$ and imposing there the heat flux $q_{2}$ and temperature $T_{2}$ leads to the desired relations between the temperatures and heat fluxes at both faces of the glass pane:

$q_{2}-q_{1}=\int_{0}^{d} \int_{0}^{\infty} q_{\mathrm{rad}, \lambda}(\xi, \lambda) \mathrm{d} \lambda \mathrm{d} \xi$

$-k\left(T_{2}-T_{1}\right)-q_{1} d=\int_{0}^{d} \int_{0}^{\eta} \int_{0}^{\infty} q_{\mathrm{rad}, \lambda}(\xi, \lambda) \mathrm{d} \lambda \mathrm{d} \xi \mathrm{d} \eta$

where the expression for the heat source term given in Eq. (3) has been substituted. In order to complete the solution to the one-dimensional absorption-conduction problem, the integrals on the right hand side of the equations need to be evaluated.

\subsection{Direct absorptances $\alpha_{e}$ and $\alpha_{e}^{\prime}$}

In order to evaluate the integrals on the right hand side of Eqs. (8) and (9) it is necessary to realize that the integration in the coordinate $x$ and in the wavelength $\lambda$ can be interchanged as the integration limits are independent of each other. By taking Eq. (8), permuting both integrals, substituting Eq. (2) for the integrand, and carrying out the integration in the space coordinate, one obtains

$$
\begin{aligned}
q_{2}-q_{1}= & \int_{0}^{\infty} \int_{0}^{d} q_{\mathrm{rad}, \lambda}(\xi, \lambda) \mathrm{d} \xi \mathrm{d} \lambda \\
= & \int_{0}^{\infty} i_{\mathrm{in}}(\lambda) \int_{0}^{d} a(\lambda) e^{-a(\lambda) \xi} \mathrm{d} \xi \mathrm{d} \lambda \\
& +\int_{0}^{\infty} j_{\mathrm{in}}(\lambda) \int_{0}^{d} a(\lambda) e^{-a(\lambda)(d-\xi)} \mathrm{d} \xi \mathrm{d} \lambda \\
= & \int_{0}^{\infty}\left[i_{\mathrm{in}}(\lambda)+j_{\mathrm{in}}(\lambda)\right]\left[1-\tau_{1}(\lambda)\right] \mathrm{d} \lambda
\end{aligned}
$$

where $\tau_{1}(\lambda)=e^{-a(\lambda) d}$ is the attenuation experienced by a ray of wavelength $\lambda$ crossing once the glass pane. Next, expressing the rays $i_{\text {in }}(\lambda)$ and $j_{\text {in }}(\lambda)$ in terms of the incoming radiations $i_{0}(\lambda)$ and $j_{0}(\lambda)$ through Eq. (1) leads to

$$
\begin{aligned}
q_{2}-q_{1}= & \int_{0}^{\infty}\left[A(\lambda) i_{0}(\lambda)+B(\lambda) j_{0}(\lambda)\right]\left[1-\tau_{1}(\lambda)\right] \mathrm{d} \lambda \\
& +\int_{0}^{\infty}\left[C(\lambda) i_{0}(\lambda)+D(\lambda) j_{0}(\lambda)\right]\left[1-\tau_{1}(\lambda)\right] \mathrm{d} \lambda \\
= & \int_{0}^{\infty}\left[\alpha(\lambda) i_{0}(\lambda)+\alpha^{\prime}(\lambda) j_{0}(\lambda)\right] \mathrm{d} \lambda
\end{aligned}
$$

where the spectral direct absorptances $\alpha(\lambda)$ and $\alpha^{\prime}(\lambda)$ given by

$$
\alpha(\lambda)=[A(\lambda)+C(\lambda)]\left[1-\tau_{1}(\lambda)\right]
$$$$
\alpha^{\prime}(\lambda)=[B(\lambda)+D(\lambda)]\left[1-\tau_{1}(\lambda)\right]
$$

have been introduced. These quantities, when multiplied by the spectral distributions of their respective incoming radiations, directly give the energy absorbed in the glass pane per unit time, unit glass surface, and unit wavelength. By defining now the direct absorptances $\alpha_{e}$ and $\alpha_{e}^{\prime}$ as the wavelength averaged counterparts of the spectral direct absorptances:

$\alpha_{e}=\frac{\int_{0}^{\infty} \alpha(\lambda) i_{0}(\lambda) \mathrm{d} \lambda}{\int_{0}^{\infty} i_{0}(\lambda) \mathrm{d} \lambda} \quad \alpha_{e}^{\prime}=\frac{\int_{0}^{\infty} \alpha^{\prime}(\lambda) j_{0}(\lambda) \mathrm{d} \lambda}{\int_{0}^{\infty} j_{0}(\lambda) \mathrm{d} \lambda}$

and the total incoming radiations $i_{0}$ and $j_{0}$ as

$i_{0}=\int_{0}^{\infty} i_{0}(\lambda) \mathrm{d} \lambda \quad j_{0}=\int_{0}^{\infty} j_{0}(\lambda) \mathrm{d} \lambda$

the final form of Eq. (8) is obtained:

$q_{2}-q_{1}=\alpha_{e} i_{0}+\alpha_{e}^{\prime} j_{0}$

This equation, which is exact, represents the overall energy balance in the glass pane: the absorbed radiation energy per unit time and unit glass surface is in equilibrium with the difference in heat fluxes flowing through the left and right faces of the glass pane.

This result coincides with the one found in the literature $[19,20,22,26]$, since the non-uniform absorption of the incoming radiation does not play a role so far, and a global energy balance of the glass pane leads to the very same result. Additionally, the definitions given for the direct absorptances $\alpha_{e}$ and $\alpha_{e}^{\prime}$ as well as for the incoming radiations $i_{0}$ and $j_{0}$ coincide with the ones found in 
the literature $[15,20,22,26]$. All this implies that the use of Eq. (15) and the computations of the direct absorptances $\alpha_{e}$ and $\alpha_{e}^{\prime}$ following Eqs. (12) and (13) pose no difficulties at all, as existing glazing simulation tools already use and compute them.

\subsection{Direct absorptance moments $\beta_{e}$ and $\beta_{e}^{\prime}$}

The next step is to carry exactly the same steps as in the previous subsection, but starting from Eq. (9). Permuting the integral in the wavelength with the two spatial integrals, substituting then Eq. (2) for the integrand, and carrying out the two integrations in the space coordinate leads to

$$
\begin{aligned}
-k\left(T_{2}-T_{1}\right)-q_{1} d= & \int_{0}^{\infty} \int_{0}^{d} \int_{0}^{\eta} q_{\mathrm{rad}, \lambda}(\xi, \lambda) \mathrm{d} \xi \mathrm{d} \eta \mathrm{d} \lambda \\
= & \int_{0}^{\infty} i_{\mathrm{in}}(\lambda) \int_{0}^{d} \int_{0}^{\eta} a(\lambda) e^{-a(\lambda) \xi} \mathrm{d} \xi \mathrm{d} \eta \mathrm{d} \lambda \\
& +\int_{0}^{\infty} j_{\mathrm{in}}(\lambda) \int_{0}^{d} \int_{0}^{\eta} a(\lambda) e^{-a(\lambda)(d-\xi)} \mathrm{d} \xi \mathrm{d} \eta \mathrm{d} \lambda \\
= & d \int_{0}^{\infty} i_{\mathrm{in}}(\lambda)\left[1+\frac{1-\tau_{1}(\lambda)}{\ln \left(\tau_{1}(\lambda)\right)}\right] \mathrm{d} \lambda \\
& -d \int_{0}^{\infty} j_{\text {in }}(\lambda)\left[\tau_{1}(\lambda)+\frac{1-\tau_{1}(\lambda)}{\ln \left(\tau_{1}(\lambda)\right)}\right] \mathrm{d} \lambda
\end{aligned}
$$

where the definition of $\tau_{1}(\lambda)$ has been used to substitute the appearance of $a(\lambda) d=-\ln \left(\tau_{1}(\lambda)\right)$ in the last expression. Next, dividing by the thickness $d$ and substituting Eq. (1) for the right and left traveling rays $i_{\text {in }}(\lambda)$ and $j_{\text {in }}(\lambda)$ leads to:

$$
\begin{aligned}
-\frac{k}{d}\left(T_{2}-T_{1}\right)-q_{1}= & \int_{0}^{\infty}\left[A i_{0}(\lambda)+B j_{0}(\lambda)\right]\left[1+\frac{1-\tau_{1}}{\ln \left(\tau_{1}\right)}\right] \mathrm{d} \lambda \\
& -\int_{0}^{\infty}\left[C i_{0}(\lambda)+D j_{0}(\lambda)\right]\left[\tau_{1}+\frac{1-\tau_{1}}{\ln \left(\tau_{1}\right)}\right] \mathrm{d} \lambda \\
= & \int_{0}^{\infty}\left[\beta(\lambda) i_{0}(\lambda)+\beta^{\prime}(\lambda) j_{0}(\lambda)\right] \mathrm{d} \lambda
\end{aligned}
$$

where the wavelength dependencies of $A(\lambda), B(\lambda), C(\lambda), D(\lambda)$, and $\tau_{1}(\lambda)$ have been omitted for the sake of clarity. In the above expression the spectral direct absorptance moments $\beta(\lambda)$ and $\beta^{\prime}(\lambda)$, given by

$$
\begin{aligned}
& \beta(\lambda)=\frac{\alpha(\lambda)}{2}+[A(\lambda)-C(\lambda)]\left[\frac{1+\tau_{1}(\lambda)}{2}+\frac{1-\tau_{1}(\lambda)}{\ln \left(\tau_{1}(\lambda)\right)}\right] \\
& \beta^{\prime}(\lambda)=\frac{\alpha^{\prime}(\lambda)}{2}+[B(\lambda)-D(\lambda)]\left[\frac{1+\tau_{1}(\lambda)}{2}+\frac{1-\tau_{1}(\lambda)}{\ln \left(\tau_{1}(\lambda)\right)}\right]
\end{aligned}
$$

have been introduced. These quantities, when multiplied by the spectral distributions of their respective incoming radiations, directly give the moment of the energy absorbed in the glass pane per unit time, unit glass surface, and unit wavelength with respect to the right face of the glass pane. By defining now the direct absorptance moments $\beta_{e}$ and $\beta_{e}^{\prime}$ as the wavelength averaged counterparts of the spectral direct absorptances moments:

$\beta_{e}=\frac{\int_{0}^{\infty} \beta(\lambda) i_{0}(\lambda) \mathrm{d} \lambda}{\int_{0}^{\infty} i_{0}(\lambda) \mathrm{d} \lambda} \quad \beta_{e}^{\prime}=\frac{\int_{0}^{\infty} \beta^{\prime}(\lambda) j_{0}(\lambda) \mathrm{d} \lambda}{\int_{0}^{\infty} j_{0}(\lambda) \mathrm{d} \lambda}$

the final form of Eq. (9) is obtained:

$-\frac{k}{d}\left(T_{2}-T_{1}\right)-q_{1}=\beta_{e} i_{0}+\beta_{e}^{\prime} j_{0}$

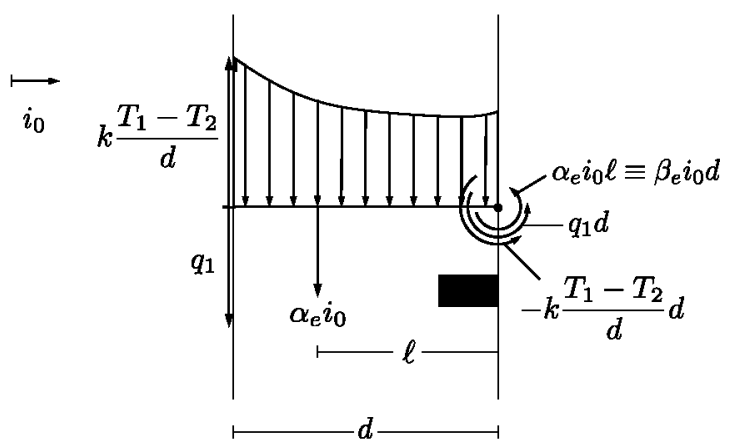

Fig. 2. Graphical representation of the balance of heat flux moments with respect to the right face of the glass pane, in which the different contributions to the balance are shown.

This equation, which again is exact, can be interpreted as a balance of heat flux moments with respect to the right face of the glass pane, where the direct absorptance moments $\beta_{e}$ and $\beta_{e}^{\prime}$ represent, in a non-dimensional way, the moment generated by the radiation absorption distribution with respect to the right face of the glass pane.

To better understand the above equation and the meaning of the direct absorptance moments, recast Eq. (20) as follows:

$0=-k \frac{T_{1}-T_{2}}{d} d+q_{1} d+\beta_{e} i_{0} d+\beta_{e}^{\prime} j_{0} d$

and assume, for the sake of clarity, that $j_{0}=0$. Fig. 2 shows a graphical representation of the resulting balance of heat flux moments. The radiation absorption that takes place across the whole glass pane leads to a resultant absorption that is equal to $\alpha_{e} i_{0}$, and whose application point is located at a distance $\ell$, unknown at this point, from the right face of the glass pane. The resulting heat flux moment with respect to the right face is then $\alpha_{e} i_{0} \ell$, which is equivalent to the heat flux moment obtained by means of the direct absorptance moment $\beta_{e}$. Thus, $\alpha_{e} i_{0} \ell \equiv \beta_{e} i_{0} d$, an equality that allows to determine, if necessary, the application point of the resultant absorption. The remaining heat fluxes in the problem also contribute to the balance of heat flux moments with respect to the right face of the glass pane, as shown in Fig. 2.

Although the defined direct absorptance moments $\beta_{e}$ and $\beta_{e}^{\prime}$ are completely new, their computation by simulation tools like WINDOW [23], VISION [24], or EnergyPlus [6] does not pose any particular difficulties, as the same results from the multiple reflections problem (the functions $A(\lambda), B(\lambda), C(\lambda)$, and $D(\lambda)$ ) and the same glazing material properties (the absorption coefficient $a(\lambda)$ ) are used as for the computation of the direct absorptances $\alpha_{e}$ and $\alpha_{e}^{\prime}$. Additionally, Eq. (20) is very similar to the equation imposed nowadays, as will be seen in Section 7, which again eases its implementation and use by existing glazing simulation tools.

\section{Two limiting cases}

To better show the connection between the direct absorptance moments $\beta_{e}$ and $\beta_{e}^{\prime}$ and the spatial distribution of radiation absorption, the present section considers two limiting cases which are very enlightening and which are also relevant for practical applications and existing approximate models for the absorption-conduction problem.

\subsection{The weakly absorbing limit}

When the considered glass pane is very thin, or when the absorption coefficient $a(\lambda)$ of the material is very small, the radiation crossing the glass pane is only weakly attenuated, so that $\tau_{1}$ is very close to unity, or alternatively: $1-\tau_{1} \ll 1$. In this limiting case, 
the expressions for the spectral direct absorptance moments given in Eq. (18) can be simplified as follows:

$\beta(\lambda) \simeq \frac{\alpha(\lambda)}{2} \quad \beta^{\prime}(\lambda) \simeq \frac{\alpha^{\prime}(\lambda)}{2}$

Analogous relations hold between the direct absorptance moments and the direct absorptances:

$\beta_{e}=\frac{\alpha_{e}}{2} \quad \beta_{e}^{\prime}=\frac{\alpha_{e}^{\prime}}{2}$

To help interpret this result, consider the expression of Bouguer's Law given in Eq. (2). In the present limit of weakly absorbing glass panes this expression simplifies to

$q_{\mathrm{rad}, \lambda}(x, \lambda) \simeq a(\lambda)\left[i_{\text {in }}(\lambda)+j_{\text {in }}(\lambda)\right]$

It can be seen that in this case the energy absorption per unit time, unit volume, and unit wavelength no longer depends on the spatial coordinate $x$, and is therefore uniform throughout the glass pane. In that case, its moment with respect to the right face of the glass pane is equal to the total absorption times the lever arm of its centroid, which is the half-width of the glass pane. This is exactly what is expressed in Eq. (23), but in non-dimensional form.

\subsection{The strongly absorbing limit}

Consider now the case of a strongly absorbing glass pane, either because it is very thick or because its absorption coefficient $a(\lambda)$ is very large. Since the radiation crossing the glass pane is strongly attenuated, $\tau_{1} \ll 1$. Additionally, no multiple reflections occur in this case, and the right traveling radiation $i_{\text {in }}$ is solely due to $i_{0}$ and the left traveling radiation $j_{\text {in }}$ is solely due to $j_{0}$. Hence, $B(\lambda) \simeq 0$ and $C(\lambda) \simeq 0$. Taking into account both facts, the expressions given in Eq. (18) for the spectral direct absorptance moments are simplified as follows:

$$
\begin{aligned}
& \beta(\lambda) \simeq \frac{\alpha(\lambda)}{2}+\frac{A(\lambda)}{2} \\
& \beta^{\prime}(\lambda) \simeq \frac{\alpha^{\prime}(\lambda)}{2}-\frac{D(\lambda)}{2}
\end{aligned}
$$

Comparing these expressions with those given in Eq. (12) for the spectral direct absorptances leads to:

$\beta(\lambda)=\alpha(\lambda) \quad \beta^{\prime}(\lambda)=0$

and again the same relations hold between the direct absorptance moments and the direct absorptances:

$\beta_{e}=\alpha_{e} \quad \beta_{e}^{\prime}=0$

This result indicates that the total absorption of the incoming radiation is taking place very close to the faces of the glass pane. In the case of the radiation $i_{0}$ incident from the left, the absorptance moment with respect to the right face of the glass pane is maximum, since the lever arm is equal to the total thickness of the glass pane. For the radiation $j_{0}$ incident from the right the absorptance moment with respect to the right face of the glass pane is zero, since the lever arm is in that case zero.

\subsection{The general case}

In the general case, the absorption of radiation takes place in a non-uniform and non-localized manner across the whole glass pane's thickness, as represented in Fig. 2. In this case, the values attained by the direct absorptance moments $\beta_{e}$ and $\beta_{e}^{\prime}$ are in between the values attained in the two limiting cases presented above:

$\frac{\alpha_{e}}{2}<\beta_{e}<\alpha_{e}, \quad 0<\beta_{e}^{\prime}<\frac{\alpha_{e}^{\prime}}{2}$
This can be verified with the results presented in Section 8 for the overall spectral and thermal response of a single glass pane of different glass types and thicknesses.

\section{Extension to coated glass panes}

The results of the previous sections are only valid for uncoated glass panes. However, the use of coatings to modify the spectral characteristics of glass panes, and with it of more complex glazings, is growing rapidly, and their influence on the thermal behavior of glass panes is important due to their localized absorption of radiation.

In this section, the above formulation is extended to take into account the effects of applied coatings in an exact way. Consider the most general case shown in Fig. 3, where a single glass pane has coatings applied on its two surfaces. The depicted coated glass represents either a single glazing or a glass pane that is part of a more complex glazing configuration. Since the thickness of the applied coatings is much smaller than the thickness of the glass pane, their presence is modeled as complex air-glass interfaces which absorb radiation and whose temperatures are constant. Thus, it is now necessary to distinguish between the heat fluxes at the left and at the right of the surfaces of the glass pane, as indicated in the figure.

The fraction of radiation absorbed at each of the coatings is obtained from the solution of the multiple reflections problem, now including the spectral effects of the coatings, by using any of the methods that belong to the second group of main buildings blocks of energy models for glazings $[13,15,34]$. Once the spectral direct absorptances at the coatings are known, direct absorptances are obtained by evaluating integrals analogous to those given in Eq. (13).

The described steps to obtain the direct absorptances at the coatings are already done by existing glazing simulation tools like WINDOW [23] or VISION [24], thus no new difficulties are introduced at this point with respect to what exists nowadays.

Once the radiation absorption at each of the coatings is known, use of the energy conservation equation in integral form applied at each of the surfaces of the glass pane is made. This leads to the following interface energy balances, which express that the absorbed radiation energies per unit time and unit glass surface are in equilibrium with the differences in heat fluxes flowing through the left and the right faces of the interfaces:

$$
\begin{aligned}
& q_{1^{+}}-q_{1^{-}}=\alpha_{c 1} i_{0}+\alpha_{c 1}^{\prime} j_{0} \\
& q_{2^{+}}-q_{2^{-}}=\alpha_{c 2} i_{0}+\alpha_{c 2}^{\prime} j_{0}
\end{aligned}
$$

where $\alpha_{c 1}, \alpha_{c 1}^{\prime}, \alpha_{c 2}$, and $\alpha_{c 2}^{\prime}$ are the direct absorptances of the coatings obtained from the steps described in the previous paragraphs.

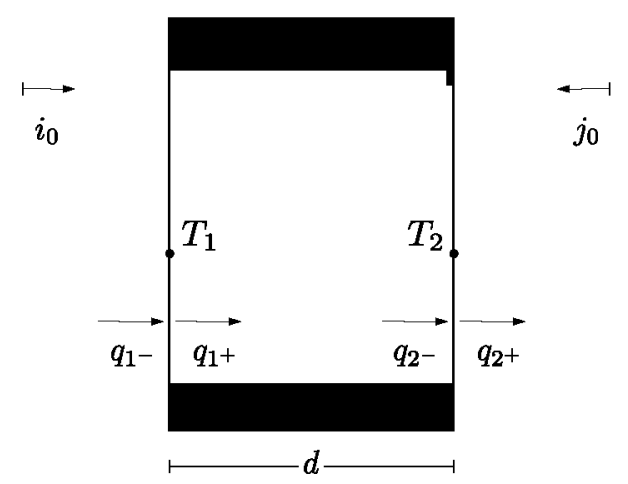

Fig. 3. Sketch of the thermal problem that arises from the interaction of the incoming radiations $i_{0}$ and $j_{0}$ with a glass pane of thickness $d$ and thermal conductivity $k$ with coatings applied on its two surfaces. 
Once the equations for the complex interfaces are derived, the thermal behavior of the glass pane itself is described by Eqs. (15) and (20), which represent now relations between the heat fluxes $q_{1+}$ and $q_{2-}$ and the surface temperatures $T_{1}$ and $T_{2}$ :

$$
\begin{array}{ll}
q_{2^{-}}-q_{1^{+}} & =\alpha_{e} i_{0}+\alpha_{e}^{\prime} j_{0} \\
-\frac{k}{d}\left(T_{2}-T_{1}\right)-q_{1+} & =\beta_{e} i_{0}+\beta_{e}^{\prime} j_{0}
\end{array}
$$

Substituting Eq. (29) into Eq. (30) and noting that $q_{1^{-}}=q_{1}$ and $q_{2^{+}}=q_{2}$, the following set of equations is obtained for the thermal behavior of coated single glass panes:

$q_{2}-q_{1}=\alpha_{\mathrm{eq}} i_{0}+\alpha_{\mathrm{eq}}^{\prime} j_{0}$

$-\frac{k}{d}\left(T_{2}-T_{1}\right)-q_{1}=\beta_{\mathrm{eq}} i_{0}+\beta_{\mathrm{eq}}^{\prime} j_{0}$

where equivalent direct absorptances and absorptance moments have been defined as follows:

$$
\begin{array}{ll}
\alpha_{\mathrm{eq}}=\alpha_{e}+\alpha_{c 1}+\alpha_{c 2} & \alpha_{\mathrm{eq}}^{\prime}=\alpha_{e}^{\prime}+\alpha_{c 1}^{\prime}+\alpha_{c 2}^{\prime} \\
\beta_{\mathrm{eq}}=\beta_{e}+\alpha_{c 1} & \beta_{\mathrm{eq}}^{\prime}=\beta_{e}^{\prime}+\alpha_{c 1}^{\prime}
\end{array}
$$

The derived equations for the thermal behavior of coated glass panes are thus analogous to the ones derived for uncoated glass panes except that alternative magnitudes for the direct absorptances and absorptance moments are used. This is very convenient, since expressions for more complex glazings can be derived without needing to worry about the presence or not of applied coatings.

Two aspects from the defined equivalent absorptances and absorptance moments shall be highlighted. First, the radiation energy absorbed at both coatings is accounted for in the equivalent direct absorptance of the coated glass pane. This is not surprising, since now the global energy balance also includes the coatings as a result of substituting the interface energy balances into the global energy balance of the glass pane alone. Second, only the absorption that takes place at the left coating contributes to the equivalent direct absorptance moments, since the lever arm of the right coating with respect to the right face of the glass pane is zero. On the contrary, the contribution of the left coating is maximum since its lever arm with respect to the right face of the glass pane is equal to the full glass pane thickness. The latter aspect regarding the equivalent direct absorptance moments is consistent with the theoretical results obtained in Section 4 for the strongly absorbing limit, where all the radiation is absorbed close to the glass pane surfaces, and therefore can be regarded as the localized absorption associated to the presence of coatings.

\section{Extension to non-normal incidence angles}

In previous sections the assumption that the incoming radiation shines normal to the glazing has been made. However, real world applications of glazings in buildings involve incidence angles which depart significantly from that. As shown by Platzer [37], not taking into account this fact when solving the spectral and thermal problem of the glass pane overpredicts the cooling needs by up to $100 \%$ and underpredicts the heating needs by up to $30 \%$. These error levels are obviously not acceptable when studying instantaneous or annual energy consumptions of individual glazings [4,5] or of whole buildings [6], requiring thus the inclusion of the non-normal incidence angle effects into the thermal models to develop.

In this aspect, the analytical solution to the one-dimensional absorption-conduction problem derived in the present work also applies to the case of non-normal incidence angles, and in fact allows an exact treatment of the resulting effects. The following three remarks need to be taken into account for that.

First, when analyzing the interaction of the incoming radiation with the glazing by means of any of the methods belonging to the second group of main buildings blocks of energy models for glazings, it is necessary to take into account the optical effects that appear due to the non-normal incidence, like the refraction of radiation or the polarization of the incoming radiation [15]. The outcome of these methods will still be the four functions $A, B, C$, and $D$ of Eq. (1), except that they will now also depend on the incidence angle $\theta$.

Second, when determining the absorption by the glass pane of the right and left traveling radiations by means of Bouguers's Law, Eq. (2), the effective absorption coefficient $a_{\text {eff }}(\lambda, \theta)=a(\lambda) / \cos \theta_{r}(\lambda)$ must be used instead, where $\theta_{r}(\lambda)$ is the refraction angle of the incoming radiation inside the considered glass pane, given by Snell's Law [35], and which depends on $\lambda$ due to the wavelength dependence of the optical properties of the material.

And third, the projection of the incoming radiation onto the normal to the glazing needs to be performed in order to obtain the absorption of radiation per unit area of glazing, which is what is required in the energy conservation equation given in Eq. (4).

All the remaining equations and expressions remain the same, except that the above mentioned remarks need to be taken into account. For instance, the direct absorptances $\alpha_{e}$ and $\alpha_{e}^{\prime}$ and the direct absorptances moments $\beta_{e}$ and $\beta_{e}^{\prime}$ will be defined by the very same equations but will depend now on the incidence angles of the incoming radiations. And the resulting global relations, which represent the exact solution to the absorption-conduction problem inside the glass pane, will be given by:

$$
\begin{array}{ll}
q_{2}-q_{1} & =\alpha_{\mathrm{eq}}\left(\theta_{i}\right) \cos \theta_{i} i_{0}+\alpha_{\mathrm{eq}}^{\prime}\left(\theta_{j}\right) \cos \theta_{j} j_{0} \\
-\frac{k}{d}\left(T_{2}-T_{1}\right)-q_{1} & =\beta_{\mathrm{eq}}\left(\theta_{i}\right) \cos \theta_{i} i_{0}+\beta_{\mathrm{eq}}^{\prime}\left(\theta_{j}\right) \cos \theta_{j} j_{0}
\end{array}
$$

where $\theta_{i}$ and $\theta_{j}$ are, respectively, the incidence angles of $i_{0}$ and $j_{0}$.

\section{Existing approximate thermal models}

The derived expressions given in Eqs. (31) and (32) represent exact relations between the heat fluxes and temperatures at both sides of the glass pane, which correctly take into account the multiple reflections phenomenon, the spectral distribution of the incoming radiation, the spectral dependence of the absorption coefficient $a(\lambda)$ of the material, the non-uniform absorption of the incoming radiation, the diffusion of heat by conduction across the glass pane, and the presence of possible coatings. In the present Section, the existing approximate models for the absorption-conduction problem are reviewed, and their merits and limitations shown.

Different approaches can be found in the literature to model or approximate the absorption-conduction problem inside the glass pane. The most extended ones employ the global energy balance derived in Eq. (31), but substitute the heat flux moment balance given in Eq. (32) by an alternative relation. For example, the constant temperature approximation $[21,22,34]$, which neglects the thermal conduction problem inside the glass pane, implicitly uses the condition $T_{1}=T_{2}$ instead. And the uniform absorption approximation $[6,20,23,24,26,27]$, which neglects the non-uniform nature of the radiation absorption process, uses the following equation instead:

$$
-\frac{k}{d}\left(T_{2}-T_{1}\right)-q_{1}=\frac{\alpha_{\mathrm{eq}}}{2} i_{0}+\frac{\alpha_{\mathrm{eq}}^{\prime}}{2} j_{0}
$$

which coincides with the results presented in Section 4 for the weakly absorbing limit. The latter approximation is nowadays the most extended one and is employed by most of the simulation tools in use $[6,23,24]$. Its replacement by the proposed model is straightforward thanks to the high degree of similarity that exists between Eqs. (35) and (32). 
In recent years more advanced models have been developed which take into account the non-uniform absorption of the incoming radiation. All of them are based on the direct solution of the energy conservation equation given in Eq. (4). However, most of them make important simplifications in how the incoming radiation is absorbed by the glass pane.

Almost all of the reviewed models neglect, completely or in part, the spectral dependence of the absorption coefficient $a(\lambda)$ of the material as well as the spectral distributions of the incoming radiations $i_{0}(\lambda)$ and $j_{0}(\lambda)[1-3,18,28-33]$. These are important simplifications taking into account that the absorption coefficient $a(\lambda)$ of glass typically varies two orders of magnitude over the wavelength range of the solar spectrum, and that the solar radiation is also not constant over that wavelength range.

Another common simplification is to neglect, completely or in part, the multiple reflections phenomenon that arises due to the presence of multiple material interfaces [1-3,28-33]. This is also an important source of errors since the multiple reflections typically increase by $3-4 \%$ the amount of radiation absorbed by a single glass pane.

Only Rosenfeld [16] solves the complete one-dimensional absorption-conduction problem including all the relevant physical phenomena. In his derivation, a new parameter $\gamma$ is introduced which represents the normalized average thermal resistance between the outer surface of the glass pane and the locations inside the glass pane where the radiation is absorbed, weighted by the amount of radiation absorbed at each location. However, his approach focuses only on the determination of the so-called secondary heat transfer factor towards the inside $q_{i}[21,22]$. This makes it difficult to extend his results for inclusion in advanced glazing simulation tools like WINDOW [23] or VISION [24], or in future revisions of the advanced international standard ISO 15099 [20], where global relations between surface temperatures and heat fluxes are required.

In contrast, the approach followed in the present work directly leads to the global relations required by these simulation tools and standards, namely Eqs. (31) and (32). Additionally, the parameter $\gamma$ can be obtained in terms of the direct absorptance moment $\beta_{e}$ by comparing the results given by the two approaches for the thermal response of a single glass pane:

$\gamma=1-\frac{\beta_{e}}{\alpha_{e}}$,

showing that both approaches are indeed equivalent, although they express the solution in different ways.

\section{Comparison with existing thermal models}

To have the exact analytical solution to a physical problem, like it happens in the present case, allows the precise assessment of the accuracy of existing approximate thermal models. This is done in the present Section by performing a numerical comparison of the two most extended approximate models, the constant temperature and the uniform absorption models, with the derived analytical solution. The problem of the single glass pane shown in Fig. 4 is used for the comparison, for which its overall spectral and thermal response is obtained using the two approximate thermal models and the analytical solution.

Since the aim of the present Section is to quantify the errors introduced by existing approximate thermal models for the absorption-conduction problem, and not the computation of real world performance indexes of single glazings, simple models will be used for the remaining three main buildings blocks that comprise the complete energy model for the single glazing.

First, the exterior and interior conditions are modeled as follows. The spectral distribution $i_{0}(\lambda)$ of the incoming radiation from

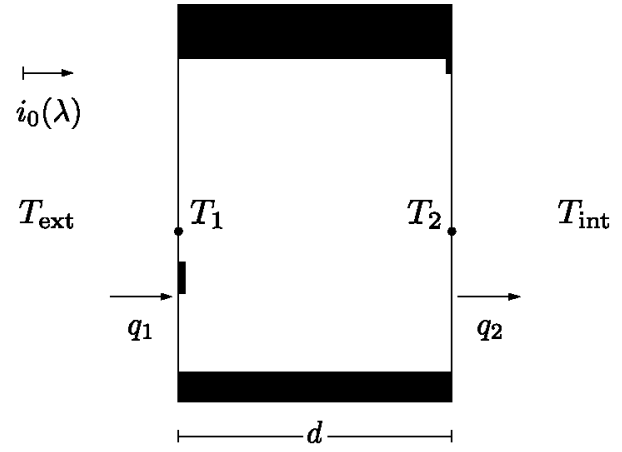

Fig. 4. Spectral and thermal problem of a single glazing of thickness $d$ and thermal conductivity $k$ exposed to the incoming radiation from the left $i_{0}(\lambda)$ and exposed to the exterior and interior environments at temperatures $T_{\mathrm{ext}}$ and $T_{\mathrm{int}}$, respectively.

outside the building is taken to be the hemispherical solar spectral irradiance defined in the international standard ISO 9845-1 [38], and its incidence angle is considered to be normal to the glazing in compliance with the standard reference conditions specified in the actual standards in force $[21,22]$. On the other side, the radiation shining from inside the building is considered to be zero: $j_{0}=0$.

Second, the interaction of the incoming radiation and the single glass pane, with the resulting multiple reflections problem to solve, is addressed by using the ray tracing method proposed by Rubin in [15]. The spectral properties of the glass pane material required by the chosen method are the ones presented by Rubin in [39]. Known the functions $A(\lambda), B(\lambda), C(\lambda)$, and $D(\lambda)$, the spectral direct absorptance $\alpha(\lambda)$ and spectral direct absorptance moment $\beta(\lambda)$ are obtained from Eqs. (12) and (18), respectively, while their overall counterparts $\alpha_{e}$ and $\beta_{e}$ are obtained by evaluating numerically the integrals present in Eqs. (13) and (19).

Finally, the heat transfer between the glass pane surfaces and the exterior and interior environments through natural/forced convection and thermal radiation is modeled using Newton's Law of Cooling and the constant heat transfer coefficients specified in the actual standards in force $[40,41]$, namely $h_{\text {ext }}=23 \mathrm{~W} /\left(\mathrm{m}^{2} \mathrm{~K}\right)$ and $h_{\text {int }}=8 \mathrm{~W} /\left(\mathrm{m}^{2} \mathrm{~K}\right)$. Although this a very rude approximation to the ongoing non-linear physical processes, it is important to remember that the aim of the analysis is not the computation of real world performance indexes of single glazings, but the quantification of the errors associated with the different models for the absorption-conduction problem inside the glass pane.

For real world applications, better models for the convective and radiative heat transfer processes are required. Then, the heat transfer coefficients $h_{\text {ext }}$ and $h_{\text {int }}$ will depend, in non-linear ways, on the glass surface temperatures as well as on the exterior and interior conditions, and therefore will no longer be constant. See for example the models used in ISO 15099 [20] or EnergyPlus [6], where experimental correlations for the heat exchange through natural and forced convection are used, and the heat exchange through thermal radiation is treated in a detailed way.

With all the above indications, the following system of four algebraic equations has to be solved:

$$
\begin{array}{ll}
q_{2}-q_{1} & =\alpha_{e} i_{0} \\
-\frac{k}{d}\left(T_{2}-T_{1}\right)-q_{1} & =\beta_{e} i_{0} \\
q_{1} & =h_{\text {ext }}\left(T_{\text {ext }}-T_{1}\right) \\
q_{2} & =h_{\text {int }}\left(T_{2}-T_{\text {int }}\right)
\end{array}
$$

where the first two are the solution to the absorption-conduction problem inside the glass pane derived in the present work and the last two are the heat transfer models between the glazing and the exterior and interior environments, which are at the temperatures 
$T_{\text {ext }}$ and $T_{\text {int }}$, respectively. In the present analysis, where constant values for the heat transfer coefficients are assumed, the above system of equations is linear and can be solved analytically. However, for real world applications, it has to be solved iteratively and numerically due to the temperature dependence of the heat transfer coefficients.

Independently of the complexity of the model for the heat transfer coefficients, the above system of equations can always be solved formally for the thermal heat flux towards the interior of the building, which coincides with $q_{2}$ for the case of a single glazing. The resulting expression can be written as follows:

$q_{2}=U\left(T_{\text {ext }}-T_{\text {int }}\right)+q_{i} i_{0}$

where $U$ is the so-called thermal transmittance of the glazing [40] and $q_{i}$ is the so-called secondary heat transfer factor of the glazing towards the inside [21], both given, for the case of a single glass pane, by:

$$
\begin{aligned}
U & =\left[\frac{1}{h_{\mathrm{ext}}}+\frac{d}{k}+\frac{1}{h_{\mathrm{int}}}\right]^{-1} \\
q_{i} & =\frac{\left[\frac{1}{h_{\mathrm{ext}}}+\frac{d}{k}\right] \alpha_{e}-\frac{d}{k} \beta_{e}}{\frac{1}{h_{\mathrm{ext}}}+\frac{d}{k}+\frac{1}{h_{\mathrm{int}}}}
\end{aligned}
$$

The non-uniform absorption effects show up in two different ways. First, directly through the direct absorptance moment $\beta_{e}$ that appears in the secondary heat transfer factor $q_{i}$. Second, indirectly through the heat transfer coefficients $h_{\text {ext }}$ and $h_{\text {int }}$, but only when these depend on the surface temperatures of the glass pane and the problem needs to be solved iteratively. Although both ways could in principle be equally important, numerical sensitivity studies and the conclusions by Wright in [25] clearly indicate that the indirect effect is significantly less important than the direct effect.

It is of interest to compare the derived expression for $q_{i}$ with the ones obtained from the constant temperature and uniform absorption approximations. The first one is obtained by letting $k / d \rightarrow \infty$, which is equivalent to neglecting the thermal conduction problem inside the glass pane, and the second one by setting $\beta_{e}=\alpha_{e} / 2$, as inferred from comparing Eq. (35) with Eq. (32):

$q_{i, \mathrm{cons}}=\frac{\left[\frac{1}{h_{\mathrm{ext}}}\right] \alpha_{e}}{\frac{1}{h_{\mathrm{ext}}}+\frac{1}{h_{\mathrm{int}}}}$

$q_{i, \text { unif }}=\frac{\left[\frac{1}{h_{\text {ext }}}+\frac{1}{2} \frac{d}{k}\right] \alpha_{e}}{\frac{1}{h_{\text {ext }}}+\frac{d}{k}+\frac{1}{h_{\text {int }}}}$

In both cases, the errors introduced into the numerator of $q_{i}$ are of order $d / k$, which is small compared to $1 / h_{\text {ext }}$ as long as the glass panes are sufficiently thin. In order to quantify these errors, a set of test cases is defined, which includes three different glass types (low-iron glass, standard glass, and green mass-colored glass) and three different glass pane thicknesses $(4,8$, and $12 \mathrm{~mm}$ ). This selection of glass types and thicknesses covers the most common range of glass panes employed in single glazings as well as more complex glazing configurations, being the low-iron glass a very low absorbing one while the green mass-colored glass a strongly absorbing one. The spectral properties of the different glass types are taken from Rubin [39], while the thermal conductivity of all of them is taken to be $k=1 \mathrm{~W} /\left(\mathrm{m}^{2} \mathrm{~K}\right)$ in compliance with the standard reference conditions defined in EN 673 [40] and ISO 101292 [41].

\subsection{Uncoated single glass panes}

The resulting values for the direct absorptance $\alpha_{e}$, the direct absorptance moment $\beta_{e}$, and the secondary heat transfer factor towards the inside $q_{i}$ are given in Table 1 for the case of uncoated single glass panes. As expected, all three values increase with increasing glass pane thicknesses and absorption coefficients of the material. Also shown in Table 1 are the errors associated to the constant temperature approximation and the uniform absorption approximation, which are defined as follows:

$E_{\mathrm{cons}}=\frac{q_{i, \mathrm{cons}}-q_{i}}{q_{i}} \quad E_{\mathrm{unif}}=\frac{q_{i, \mathrm{unif}}-q_{i}}{q_{i}}$

For both approximations, the errors increase with increasing thickness of the glass pane. However, while the uniform absorption approximation presents growing errors with increasing values of the absorption coefficient of the material, the constant temperature approximation evolves in the opposite direction. The reason is that increasing values of the absorption coefficient lead to larger values of $\beta_{e}$, as shown in the analysis of the strongly absorbing limit. This makes the numerator of Eq. (40) more and more accurate. On the contrary, the numerator of Eq. (41) departs more and more from the exact solution given by Eq. (39).

Comparing now the approximations among themselves, the uniform absorption approximation clearly leads to more accurate results, with errors which in most cases are an order of magnitude lower than the ones given by the constant temperature approximation. However, for thick and mass-colored single glass panes both approximations perform equal because of the described opposite tendencies of the errors with increasing absorption coefficients of the material. For thin low-iron glass panes, the uniform absorption approximation leads to errors of about $0.1 \%$, while for thick mass-colored glass panes the errors reach $3.5 \%$. This result is in accordance with the conclusions drawn in Section 4, where the uniform absorption approximation naturally emerges from the limit of weakly absorbing glass panes, and is therefore more accurate for those types of glass panes.

Worth to mention is that the constant temperature approximation always underpredicts the value of the secondary heat transfer factor $q_{i}$, while the uniform absorption approximation always overpredicts it. This is a direct consequence of the fact that $\alpha_{e} / 2<\beta_{e}<\alpha_{e}$, as pointed out in Section 4. Hence, the numerator of the exact expression for $q_{i}$ given in Eq. (39) always lies in between the values of the numerators of the expressions given in Eqs. (40) and (41). Therefore, the results given by the two approximations can be used as lower and upper bounds for the exact solution.

Of interest for the thermal analysis of buildings is also the total heat flux $q$ that crosses the glazing and which also includes the fraction of solar radiation directly transmitted through the glazing. To obtain the transmitted fraction, first the spectral direct transmittance $\tau(\lambda)$ of the glazing is obtained from the solution of the multiple reflections problem, and then its overall counterpart $\tau_{e}$ is computed through integrals analogous to those given in Eq. (13). Then:

$q=q_{2}+\tau_{e} i_{0}=U\left(T_{\mathrm{ext}}-T_{\mathrm{int}}\right)+g i_{0}$

where $g=\tau_{e}+q_{i}$ is the so-called solar factor of the glazing [21]. Table 1 presents values for the direct transmittance $\tau_{e}$ and the solar factor $g$ of the different considered single glass panes. The same comments made before for the secondary heat transfer factor $q_{i}$ are also applicable to the solar factor $g$, except that the errors are now lower. This is because the direct transmittance $\tau_{e}$ weights more than $q_{i}$ in the solar factor and its value is independent of the model used for the absorption-conduction problem inside the glass pane. For thin low-iron glass panes, the uniform absorption approximation leads to errors in the solar factor which are negligible, while for thick mass-colored glass panes the errors reach $1.2 \%$, an acceptable error level for many practical applications. 
Table 1

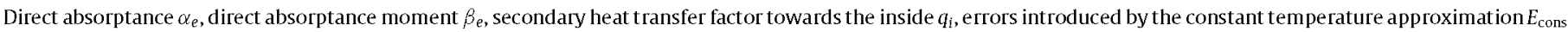

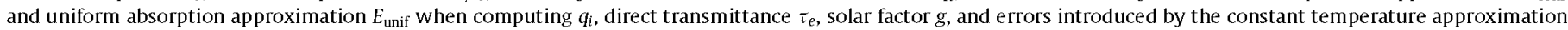
$E_{\text {cons }}$ and uniform absorption approximation $E_{\text {unif }}$ when computing $g$, for three different glass types and three different glass pane thicknesses $d$.

\begin{tabular}{|c|c|c|c|c|c|c|c|c|c|c|}
\hline & $d$ & $\alpha_{e}$ & $\beta_{e}$ & $q_{i}$ & $E_{\text {cons }}$ & $E_{\text {unif }}$ & $\tau_{e}$ & $g$ & $E_{\text {cons }}$ & $E_{\text {unif }}$ \\
\hline \multirow{3}{*}{ Low-iron glass } & $4 \mathrm{~mm}$ & 0.027 & 0.014 & 0.007 & $-20.7 \%$ & $0.6 \%$ & 0.894 & 0.901 & $-0.16 \%$ & $<0.01 \%$ \\
\hline & $8 \mathrm{~mm}$ & 0.051 & 0.026 & 0.014 & $-39.0 \%$ & $1.9 \%$ & 0.871 & 0.885 & $-0.61 \%$ & $0.03 \%$ \\
\hline & $12 \mathrm{~mm}$ & 0.075 & 0.039 & 0.020 & $-55.4 \%$ & $3.5 \%$ & 0.849 & 0.869 & $-1.30 \%$ & $0.08 \%$ \\
\hline \multirow{3}{*}{ Standard glass } & $4 \mathrm{~mm}$ & 0.105 & 0.054 & 0.028 & $-19.7 \%$ & $1.6 \%$ & 0.821 & 0.849 & $-0.64 \%$ & $0.05 \%$ \\
\hline & $8 \mathrm{~mm}$ & 0.190 & 0.101 & 0.051 & $-35.7 \%$ & $5.3 \%$ & 0.741 & 0.792 & $-2.29 \%$ & $0.34 \%$ \\
\hline & $12 \mathrm{~mm}$ & 0.261 & 0.142 & 0.071 & $-48.6 \%$ & $10.7 \%$ & 0.674 & 0.744 & $-4.63 \%$ & $1.02 \%$ \\
\hline \multirow{3}{*}{ Green glass } & $4 \mathrm{~mm}$ & 0.341 & 0.190 & 0.089 & $-16.3 \%$ & $5.1 \%$ & 0.598 & 0.688 & $-2.12 \%$ & $0.66 \%$ \\
\hline & $8 \mathrm{~mm}$ & 0.518 & 0.311 & 0.137 & $-24.2 \%$ & $17.3 \%$ & 0.428 & 0.565 & $-5.86 \%$ & $4.20 \%$ \\
\hline & $12 \mathrm{~mm}$ & 0.623 & 0.397 & 0.165 & $-26.1 \%$ & $34.6 \%$ & 0.326 & 0.492 & $-8.78 \%$ & $11.61 \%$ \\
\hline
\end{tabular}

\subsection{Coated single glass panes}

The expressions obtained in the preceding subsection are directly applicable to the analysis of coated single glass panes by just employing the equivalent direct absorptance $\alpha_{\mathrm{eq}}$ and equivalent direct absorptance moment $\beta_{\text {eq }}$ defined in Eq. (33).

To compute these quantities, the direct absorptances at the coatings need to be obtained first, namely $\alpha_{c 1}$ if the coating is applied on the left face of the glass pane and $\alpha_{c 2}$ if it is applied on the right face. As already explained in Section 5 , the direct absorptances of the coatings are obtained from the solution of the multiple reflections problem and the computation of integrals analogous to those given in Eq. (13). The spectral properties of the coatings, required for the solution of the multiple reflections problem, are obtained from solving the inverse problem defined in Appendix A.2.1 of the actual standards EN 410 [21] and ISO 9050 [22]. The required experimental measurements are performed with a Perkin-Elmer Lambda 950 spectrophotometer.

Two different coatings are considered in the present work, both from the glass manufacturer Guardian Industries: a weakly absorbing one (Guardian SunGuard E-Performance) and a strongly absorbing one (Guardian SunGuard Solar Silver 20). Both coatings are going to be applied on a $6 \mathrm{~mm}$ thick low-iron glass pane, so that the effects of the localized absorption due to the coatings are better highlighted. Although the Guardian E-Performance coating has also a low emissivity, this fact is not taken into account in the present analysis so that both coatings are compared under the same conditions. This means that the values of $h_{\text {ext }}$ and $h_{\text {int }}$ remain unchanged.

Table 2 shows the computed values for the two considered coatings. The position of the coating on the glass pane is indicated in the table by a vertical bar |, so that both possibilities are studied: applied on the left face of the glass pane and on the right face of the glass pane. The case without applied coatings is also included in the table for comparison purposes.

The first thing to note is that none of the two approximations excels with respect to the other in terms of errors, being sometimes the constant temperature approximation the better one and sometimes the uniform absorption approximation the better one. The second thing to note is that errors are much higher when coatings are present, reaching almost $8.5 \%$ for the secondary heat transfer factor towards the inside $q_{i}$ and $4 \%$ for the solar factor $g$. This is caused by the strongly localized nature of the absorption at the coating, and less by the amount of absorbed radiation. This is confirmed by the results shown in the table, where both types of coatings present similar errors for $q_{i}$, but have very different equivalent direct absorptances $\alpha_{\mathrm{eq}}$.

However, things start to differ when looking at the solar factor $g$, where computations done with the low absorbing Guardian E-Performance coating present errors five times lower than those done with the more absorbing Guardian Silver 20. The reason is that the magnitudes of $q_{i}$ are very different among the two coatings, and so their relevance in the solar factor.

Another interesting outcome is the significant differences in terms of results that are observed between the two possible positions of the coating. Two reasons exist for that. The first one is that coatings do not behave in the same way for radiation incoming from the air side than for radiation incoming from the glass substrate side, leading to different values for the equivalent direct absorptance $\alpha_{\text {eq }}$. The second reason is that the position of the coating significantly changes the value of the equivalent direct absorptance moment $\beta_{\text {eq. }}$. In fact, if a perfect non-absorbing glass is considered, $a(\lambda)=0$, then:

Coating on the left face: $\alpha_{\mathrm{eq}}=\alpha_{c 1}, \quad \beta_{\mathrm{eq}}=\alpha_{c 1}=\alpha_{\mathrm{eq}}$ Coating on the right face: $\alpha_{\mathrm{eq}}=\alpha_{\mathrm{c} 2}, \quad \beta_{\mathrm{eq}}=0$

These limiting cases closely resemble the results shown in Table 2, which are computed using a low absorbing low-iron glass, and the theoretical results obtained in Section 4 for the strongly absorbing limit, where all the radiation is absorbed close to the glass pane surfaces. The final effect on the secondary heat transfer factor $q_{i}$, however, is lower than on $\beta_{\text {eq }}$ since the equivalent direct absorptance moment is multiplied by $d / k$, which for thin glass panes is small compared to $1 / h_{\text {ext }}$.

Table 2

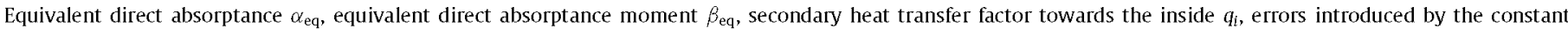

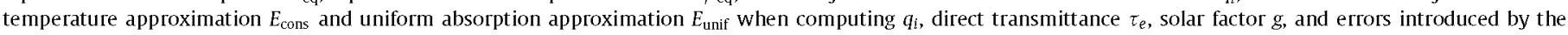

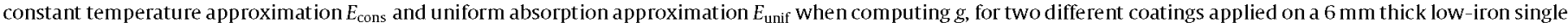
glass pane. The position of the coating on the glass pane is indicated by a vertical bar |.

\begin{tabular}{|c|c|c|c|c|c|c|c|c|c|c|}
\hline & $d$ & $\alpha_{\mathrm{eq}}$ & $\beta_{\text {eq }}$ & $q_{i}$ & $E_{\text {cons }}$ & $E_{\text {unif }}$ & $\tau_{e}$ & $g$ & $E_{\text {cons }}$ & $E_{\text {unif }}$ \\
\hline \multirow{3}{*}{ Guardian E-Performance } & $6 \mathrm{~mm}$ & 0.039 & 0.020 & 0.010 & $-30.1 \%$ & $1.2 \%$ & 0.882 & 0.892 & $-0.4 \%$ & $<0.1 \%$ \\
\hline & $6 \mathrm{~mm}$ & 0.152 & 0.146 & 0.038 & $29.5 \%$ & $62.7 \%$ & 0.460 & 0.498 & $2.3 \%$ & $4.8 \%$ \\
\hline & $61 \mathrm{~mm}$ & 0.184 & 0.028 & 0.051 & $-73.0 \%$ & $-43.1 \%$ & 0.460 & 0.511 & $-7.3 \%$ & $-4.3 \%$ \\
\hline \multirow{3}{*}{ Guardian Silver 20} & $6 \mathrm{~mm}$ & 0.039 & 0.020 & 0.010 & $-30.1 \%$ & $1.2 \%$ & 0.882 & 0.892 & $-0.4 \%$ & $<0.1 \%$ \\
\hline & $16 \mathrm{~mm}$ & 0.494 & 0.491 & 0.123 & $34.7 \%$ & $68.0 \%$ & 0.167 & 0.290 & $14.7 \%$ & $28.9 \%$ \\
\hline & $6 \mid \mathrm{mm}$ & 0.533 & 0.025 & 0.150 & $-84.8 \%$ & $-55.3 \%$ & 0.167 & 0.317 & $-40.2 \%$ & $-26.2 \%$ \\
\hline
\end{tabular}


In summary, if for uncoated single glass panes the existing approximate models for the absorption-conduction problem work sufficiently good for most practical applications, the higher errors encountered for coated single glass panes, especially for highly absorbing ones, not always render them as suitable. Thus, more accurate models for the absorption-conduction problem like the one proposed in the present work are convenient, especially when taking into account its low additional complexity and extremely similar structure when compared to the existing approximate ones, which eases its adoption and implementation into existing glazing simulation tools and standards.

\section{Conclusions}

In the present work the analytical solution to the onedimensional absorption-conduction problem that arises inside single glass panes exposed to solar radiation is presented. The derived solution correctly takes into account all the relevant physical phenomena that play a role in the absorption of solar radiation by glass panes, namely the multiple reflections caused by the presence of more than one material interface, the spectral distribution of the incoming radiations, the spectral dependence of the optical properties of the materials, the presence of possible coatings, and the fact that the radiation is non-uniformly absorbed inside materials.

The obtained exact solution is comprised by two algebraic equations which relate the temperatures and heat fluxes at the faces of the glass pane with the absorbed radiation. Additionally to the well established and known direct absorptances $\alpha_{e}$ and $\alpha_{e}^{\prime}$, the derived equations introduce new spectral quantities called direct absorptance moments $\beta_{e}$ and $\beta_{e}$, and which indicate where in the glass pane is the absorption of radiation actually taking place. The derived equations are very similar to those of existing approximate thermal models, making it straightforward to substitute the latter ones by the former ones in existing simulation tools like WINDOW [23], VISION [24], or EnergyPlus [6], or in future revisions of the advanced international standard ISO 15099 [20].

The derived analytical solution is then used to assess the accuracy of two well-established approximate models for the absorption-conduction problem inside a glass pane, namely the constant temperature model and the uniform absorption model. The latter one, which is by far the most extended one, performs very good for uncoated single glazings, with error levels of almost 3.5\% for the secondary heat transfer factor and of $1.2 \%$ for the solar factor. However, in the presence of coatings, the error levels increase, reaching almost $7 \%$ for the secondary heat transfer factor and $3 \%$ for the solar factor.

Although these error levels may be acceptable for many practical applications, the easiness of replacing in glazing simulation tools the existing thermal models by Eqs. (31) and (32), and the fact that these equations represent the exact analytical solution to the absorption-conduction problem inside the glass pane, clearly advocates for their adoption.

Extension of the analysis presented above for the derivation of exact solutions in more complex glazing configurations, such as laminated glass panes or multiple glazings, is to be considered in future work. As in the present contribution, a consistent and simple formulation of the resulting equations should be sought, enabling a clear and correct physical interpretation of the outcoming results.

\section{Acknowledgements}

The authors would like to thank Professors M. Sánchez and M. Vera for insightful discussions. This work was supported by Project
ENE2008-06683-C03-02 of the Spanish Ministerio de Ciencia e Innovación.

\section{References}

[1] K.A.R. Ismail,].R. Henríquez, Modeling and simulation of a simple glass window, Solar Energy Materials and Solar Cells 80 (3) (2003) 355-374.

[2] K.A.R. Ismail, J.R. Henrí quez, Two-dimensional model for the double glass nat urally ventilated window, International Journal of Heat and Mass Transfer 48 (3-4) (2003) 461-475.

[3] Y. Fang, T. Hyde, N. Hewitt, P.C. Eames, B. Norton, Thermal performance analysis of an electrochromic vaccum glazing with low emittance coatings, Solar Energy $84(4)(2010) 516-525$

[4] J. Karlsson, B. Karlsson, A. Roos, A simple model for assessing the energy per formance of windows, Energy and Buildings 33 (7) (2001) 641-651.

[5] P.A. Waide, B. Norton, Variation of insolation transmission with glazing plane position and sky conditions, ASME Journal of Solar Energy Engineering 125 (2) (2003) 182-189.

[6] EnergyPlus Engineering Reference, Universities of Illinois and California, 2011.

[7] M. Martin, P. Berdahl, Characteristics of infrared sky radiation in the United States, Solar Energy 33 (3-4) (1984) 321-336.

[8] C.A. Gueymard, H.D. Kambezidis, Solar spectral radiation, in: T. Muneer (Ed.), Solar Radiation and Daylight Models, second ed., Elsevier ButterworthHeinemann, Oxford, UK, 2004

[9] Q.Y. Zhang, Y.J. Huang, Development of typical year weather data for Chinese locations, ASHRAE Transactions 108 (2) (2002) 1063-1075.

[10] S. Wilcox, W. Marion, Users Manual for TMY3 Data Sets, Technical Report NREL/TP-581-43156, National Renewable Energy Laboratory, Colorado, USA 2008

[11] M. Iqbal, An Introduction to Solar Radiation, Academic Press, Ontario, Canada 1983.

[12] T. Muneer, Solar Radiation and Daylight Models, second ed., Elsevier Butterworth-Heinemann, Oxford, UK, 2004.

[13] B. Harbecke, Coherent and incoherent reflection and transmission of multilayer structures, Applied Physics B 39 (3) (1986) 165-170.

[14] P. Pfrommer, K.J. Lomas, C. Seale, C.H.R. Kupke, The radiation transfer through coated and tinted glazing, Solar Energy 54 (5) (1995) 287-299.

[15] M. Rubin, K. von Rottkay, R. Powles, Window optics, Solar Energy 63 (3) (1998) $149-161$.

[16] J.L.J. Rosenfeld, On the calculation of the total solar energy transmittance for complex glazing systems, in: Proceedings of the 8th International Meeting on Transparent Insulation, Freiburg, Germany, 1996.

[17] R. Powles, D, Curčija, C. Köhler, Solar absorption in thick and multilayered glazings, in: World Renewable Energy Congress VII, Cologne, Germany, 2002.

[18] C.S. Strobel, M.O. Abadie, N. Mendes, Absorption of solar radiation in thick and multilayered glazing, in: Building Simulation 2007 Conference, Beijing, China, 2007.

[19] M. Rubin, Calculating heat transfer through windows, International Journal of Energy Research 6 (4) (1982) 341-349.

[20] ISO 15099, Thermal performance of windows, doors and shading devices, 2003

[21] EN 410, Glass in building. Determination of luminous and solar characteristics of glazing, 1998.

[22] ISO 9050, Glass in building. Determination of light transmittance, solar direct transmittance, total solar energy transmittance, ultraviolet transmittance and related glazing factors, 2003

[23] WINDOW 4.0: Documentation of Calculation Procedures, Energy and Environment Division, Lawrence Berkley Laboratory, California, USA, 1993.

[24] VISION4 Glazing System Thermal Analysis - Reference Manual, Advanced Glazing System Laboratory, Department of Mechanical Engineering, University of Waterloo, Waterloo, Canada, 1995.

[25] J.L. Wright, Summary and comparison of methods to calculate solar heat gain, ASHRAE Transactions 101 (1) (1995) 802-818.

[26] J.L. Wright, Calculating centre-glass performance indices of windows, ASHRAE Transactions 104 (1) (1999) 1230-1241.

[27] K.G.T. Hollands, J.L. Wright, C.G. Granqvist, Glazings and coatings, in: Solar Energy - The State of the Art, James \& James, London, UK, 2001.

[28] C.A.Estrada-Gasca, G. Alvarez-Garcia, P.K. Nair, Theoretical analysis of the thermal performance of chemically deposited solar control coatings, Journal of Physics D: Applied Physics 26 (8) (1993) 1304-1309.

[29] G. Alvarez, D.N. Jiménez, C.A. Estrada, Thermal performance of solar control coatings: a mathematical model and its experimental validation, Journal of Physics D: Applied Physics 31 (18) (1998) 2249-2257.

[30] G. Alvarez, J.]. Flores, C.A. Estrada, The thermal response of laminated glass with solar cont rol coating, Journal of Physics D: Applied Physics 31 (21) (1998) 3057-3065

[31] T.Y.Y. Fung, H. Yang, Study on thermal performance of semi-transparent building-integrated photovoltaic glazings, Energy and Buildings 40 (3) (2008) 341-350.

[32] F. Noh-Pat, J. Xamán, G. Âlvarez, Y. Chávez, J. Arce, Thermal analysis for a double glazing unit with and without a solar control film ( $\mathrm{SnS}-\mathrm{Cu}_{x} \mathrm{~S}$ ) for using in hot climates, Energy and Buildings 43 (2-3) (2011) 704-712

[33] F. Zanghirella, M. Perino, V. Serra, A numerical model to evaluate the thermal behaviour of active transparent facades, Energy and Buildings 43 (5) (2011) $1123-1138$ 
[34] M. Rubin, Solar optical properties of windows, International Journal of Energy Research 6 (2) (1982) 123-133.

[35] R. Siegel, J.R. Howell, Thermal radiation heat transfer, third ed., Hemisphere Publishing Corporation, Washington, USA, 1992

[36] D.K. Edwards, Solar absorption by each element in an absorber-coverglass array, Solar Energy 19(4) (1977) 401-402.

[37] W.]. Platzer, The ALTSET project: measurement of angular properties for complex glazings, in: Proceedings of the 3rd International ISES Europe Solar Conference, Copenhagen, Denmark, 2000.
[38] ISO 9845-1, Solar energy. Reference global spectral irradiance at the ground at different receiving conditions. Part 1: direct normal and hemispherical solar irradiance for air mass $1,5,1992$.

[39] M. Rubin, Optical properties of soda lime silica glasses, Solar Energy Materials 12 (4) (1985) $275-288$.

[40] EN 673, Glass in building. Determination of thermal transmittance ( $U$ value) Calculation method, 1998.

[41] ISO10292, Glass in building. Calculation of steady-state $U$ values (thermal trans mittance) of multiple glazing, 1994. 